Nouvelles perspectives en sciences sociales

Revue internationale de systémique complexe et d'études relationnelles

\title{
Récit de Soi médiatique et identitaire. La Liberté entre francisation, rentrée scolaire, discipline et éloge
}

\section{Jean Valenti}

Volume 8, numéro 2, mai 2013

Sur le thème de représentations identitaires et expressions culturelles de la francophonie canadienne à travers ses pratiques artistiques et médiatiques

URI : https://id.erudit.org/iderudit/1016470ar

DOI : https://doi.org/10.7202/1016470ar

Aller au sommaire du numéro

Éditeur(s)

Prise de parole

ISSN

1712-8307 (imprimé)

1918-7475 (numérique)

Découvrir la revue

Citer cet article

Valenti, J. (2013). Récit de Soi médiatique et identitaire. La Liberté entre francisation, rentrée scolaire, discipline et éloge. Nouvelles perspectives en sciences sociales, 8(2), 51-84. https://doi.org/10.7202/1016470ar
Résumé de l'article

Cet article porte sur la rubrique « Dans nos écoles » du journal franco-manitobain La Liberté (2006-2008). L'auteur vise à montrer que le récit de soi y explore certaines stratégies de reconnaissance identitaires au croisement de l'individuel et du social. Il insiste sur les figures de la francisation, de la rentrée scolaire et de la discipline en milieu scolaire, tout en montrant que la dialectique de soi et de l'autre y ouvre un parcours symbolique à la faveur duquel l'autre de la culture franco-manitobaine (le monde anglophone) s'énonce implicitement. À ce titre, le récit de soi, comme forme narrative, constitue un lieu de médiations et de mémoire. Afin de mieux préciser son objet de recherche, l'auteur propose d'entrée de jeu une synthèse des travaux menés sur l'identité dans quelques champs disciplinaires en sciences humaines et sociales. Cette synthèse permet non seulement de mettre en relief la complexité du concept d'identité, mais aussi quelques idées directrices sans lesquelles celui-ci demeure perméable à tous les abus conceptuels. 


\title{
Récit de Soi médiatique et identitaire. La Liberté entre francisation, rentrée scolaire, discipline et éloge ${ }^{1}$
}

\author{
Jean Valenti \\ Université de Saint-Boniface, Winnipeg
}

Nous sommes entrés dans l'époque de l'irrésistible montée des croyances identitaires ${ }^{2}$.

[...] la présence d'un autre ou des autres est aussi évidente au niveau du récit le plus intime que celle de l'individu singulier au niveau le plus englobant du récit pluriel ou collectif3.

Bien des dieux ont été mis au rencart, mais l'individu demeure obstinément, déité d'une importance considérable .

T e récit de Soi s'impose aujourd'hui à grande échelle dans notre société de consommation, voire d'hyperconsommation ${ }^{5}$. D’un simple témoignage circonstanciel à un épisode de vie

1 Nous remercions le Conseil de recherche en sciences humaines du Canada $(\mathrm{CRSH})$, dont la contribution financière a rendu possible la rédaction de cet article.

2 Jean-Claude Kaufmann, L'Invention de soi. Une théorie de lidentité, Paris, Hachette, coll. "Littératures ", 2006, [2004], p. 24.

3 Marc Augé, Les Formes de l'oubli, Paris, Payot \& Rivages, coll. " Petite bibliothèque ", 2001, p. 60.

4 Erving Goffman, Les Rites de l'interaction, trad. de l'anglais par Alain Kihm, Paris, Éditions de Minuit, coll. "Le sens commun ", 1974 [1967], p. 84.

5 Gilles Lipovetsky, Le Bonheur paradoxale. Essai sur la société d'hyperconsommation, Paris, Gallimard, coll. "Folio/essais », 2009 [2006]. 
digne d'intérêt, en passant par une confession à variante pathétique ou polémique, le récit de Soi apparaît désormais comme une activité à la portée de tous, une manière d'exercice narratif de masse, suivant la formule de Philippe Lejeune ${ }^{6}$. Longtemps réservé à une élite détentrice des prérogatives de la culture, formée aux humanités classiques et hautement consciente de ses privilèges, le récit à valeur autobiographique gagne en effet toutes les couches de la société de nos jours, au point de devenir le genre médiatique par excellence, du moins celui qui suscite le plus de passions et d'interactions de tous ordres. On ne saurait donc l'ignorer comme phénomène social d'envergure dans le cadre d'une réflexion sur les représentations médiatiques des communautés francophones minoritaires du Canada.

Comme toute forme narrative, le récit (de soi) est un lieu de médiations et de mémoire ${ }^{7}$. Il consiste à intégrer le monde de la praxis humaine dans le monde de la narration. À ce titre, il constitue une forme d'intervention singulière dans les massmédias où il assume, en plus d'une fonction informative, plusieurs fonctions à caractère résolument pragmatique: il peut émouvoir, inciter à réfléchir, convaincre, comme il peut séduire à des fins égoïstes, inspirer de la lassitude ou de l'enthousiasme. Il est même parfois difficile de trancher entre ses propensions à la manipulation ou à la bienveillance ${ }^{8}$. Dans tous les cas de figure, médiation et mémoire font du récit de soi une pratique symbolique dûment reconnue à la faveur de laquelle l'individu s'inscrit dans le social sous diverses modalités. C'est plus particulièrement l'une d'entre elles qui intéresse cet article, la modalité identitaire. Une hypothèse de recherche dessine l'horizon de notre propos: le récit de soi contribue à la formulation identitaire en médiatisant l'expé-

\footnotetext{
$6 \quad$ Philippe Lejeune, Je est un autre : l'autobiographie, de la littérature aux médias, Paris, Éditions du Seuil, coll. "Poétique », 1980, p. 24.

7 Paul Ricour, Temps et récit, vol. 1 ("L'intrigue et le récit historique "), Paris, Éditions du Seuil, coll. "L’ordre philosophique ", 1983; Temps et récit, vol. II ("La configuration dans le récit de fiction »), Paris, Éditions du Seuil, coll. "Points/essais ", 1991 [1984]; Temps et récit, vol. III ("Le temps raconté »), Paris, Éditions du Seuil, " Points/essais ", 1991 [1985].

8 Philippe Breton, La Parole manipulée, Paris, Éditions La Découverte, coll. "Essais », 2000.
} 
rience individuelle et collective selon deux logiques: la première d'entre elles insiste sur l'action rationnelle et sur la créativité des individus, elle se prolonge tout naturellement dans l'ordre d'une connaissance autoréflexive qui vise non seulement à enrayer les risques d'assimilation, mais encore à faire œuvre communautaire sur le plan identitaire; la seconde logique s'avère plus complexe à définir, plus difficile à cerner également, mais elle n'en est pas moins présente, fût-ce de manière implicite: elle concerne les rapports entre le Soi et l'Autre, elle met en jeu une dialectique qui permet notamment de comprendre que tous les motifs de l'engagement communautaire ( "lutte », " combat » ou " résistance identitaire ") impliquent, aussi bien au niveau de l'argumentaire que de l'image de soi, le fait anglophone. C'est aussi le cas en ce qui concerne la question de la " francisation ", on aura l'occasion de le montrer plus bas. Aussi l'Autre de la culture francophone au Manitoba (l'un de ses Autres...) trouvet-il droit de cité dans le discours et sur la place publique. Ces deux logiques sont complémentaires en ce sens qu'elles contribuent à la formation d'une pratique symbolique mettant en valeur un savoir-être identitaire qui s'exprime suivant les termes d'un devoir-être collectif.

Les livraisons de novembre 2005 à janvier 2010 de la rubrique "Dans nos écoles » du journal franco-manitobain La Liberté constituent notre corpus de référence. Mais nous nous en tiendrons aux années 2006-2008 pour les fins de cet article, car nous $\mathrm{y}$ trouvons les thématisations que nous souhaitons passer au crible de l'analyse. Quelles représentations de soi (des FrancoManitobains) y retrouve-t-on sous le rapport de la francisation, de la rentrée scolaire, de la discipline et de l'éloge? Quelles formulations de l'identitaire francophone en milieu minoritaire y lit-on? Le sous-titre de cette rubrique: "Apprendre et grandir ensemble " souligne à la fois l'importance de la pédagogie et celle du vivre ensemble, l'un impliquant l'autre à divers titres comme on ne manquera pas de le souligner plus bas. En outre, cette rubrique journalistique comporte une mention à teneur générique. En effet, les articles se présentent comme autant de 
" publi-reportages ». Cela revient à dire qu'il y s'agit aussi bien d'informer le lectorat par le biais de "reportages " que d'utiliser les pages de La Liberté à des fins de publicité sociétale pour la Division scolaire franco-manitobaine. Les représentations de soi se retrouvent dans ces deux grandes fonctions de la rubrique "Dans nos écoles ». S'il ne s'agira ici pas de tracer une ligne de démarcation entre les énoncés informatifs et publicitaires dans le cadre de notre hypothèse de travail, nous entendons en revanche tirer parti de ces deux fonctions pleinement assumées par notre corpus de référence.

Divisé en trois parties, cet article propose d'abord une synthèse du concept d'identité dans quelques champs disciplinaires en sciences humaines et sociales. Sans aucunement prétendre à l'exhaustivité, souligner quelques repères historiques et méthodologiques ne sera pas négligeable, avant de chercher à savoir si les recherches en sciences humaines sur l'identité se rencontrent à un certain niveau ou si au contraire cette notion y constitue une véritable catégorie fourre-tout, une manière de faux problème persistant, sinon une vaste illusion ${ }^{9}$. Ce parcours permettra non seulement de signaler la complexité d'une notion, mais aussi quelques lignes de force de la recherche en sciences humaines sur l'identité, quelques repères-phares en effet sans lesquels la compréhension de celle-ci demeure perméable à tous les abus conceptuels. Ensuite, il s'agira d'expliquer la nature et les fonctions du récit de Soi exemplaire tel qu'il prend forme dans le corpus de référence dans la perspective de la narrativité et des rapports implicites entre le Soi et l'Autre; des exemples des deux logiques discursives à l'œuvre seront proposés dans le cadre de la francisation, de la rentrée scolaire 2008 et de la discipline en milieu scolaire. Enfin, quelques exemples de titres des articles de la rubrique "Dans nos écoles " permettront de formuler des conclusions plus générales sur la configuration d'un Nous communautaire.

9 Jean-François Bayart, L'Illusion identitaire, Paris, Fayard, coll. « L'espace du politique ", 1996. 


\section{Petit itinéraire au pays de l'identité}

À l'heure où les identités se conjuguent désormais au pluriel, où les relations interculturelles se multiplient à un rythme inédit, où les économies de marché outrepassent leurs frontières, tout en imposant leurs biens et services accompagnés de leur capital symbolique, le problème millénaire de la permanence et du changement nous interpelle plus que jamais. On peut le considérer comme la matrice générale au sein de laquelle s'énoncent les divers questionnements sur l'identité. La réflexion contemporaine inaugurée notamment par Paul Ricœur autour de la mêmeté, de l'ipséité, de la mémoire et de la reconnaissance ne passe pas sous silence le legs gréco-romain ${ }^{10}$. Si Parménide et Héraclite demeurent les premières grandes références classiques, on peut aussi citer à ce chapitre les présocratiques et les sophistes ${ }^{11}$, la rhétorique ancienne et les questions relatives à l'èthos, aux usages et aux valeurs de la polis ${ }^{12}$. À vrai dire, il n'est guère d'investigation en philosophie qui ne se consacre à définir l'identité sous le rapport de la permanence et du changement, selon telle répartition du savoir, tel objet de connaissance, depuis l'abstraction logique imperméable aux influences externes jusqu'aux incursions dans les domaines connexes de l'empirie, de l'action et du langage ordinaire. Par exemple, le vaste domaine de la philosophie pratique et existentielle présente un panorama si diversifié en développements de tous ordres sur l'identité que les doctrines les plus célèbres du passé (Platon, Aristote, Spinoza, Hume, Kant, Hegel) côtoient à tel ou tel titre celles d'un présent relativement récent (utilitarisme de Mill, décisionnisme de Weber, vitalisme de Bergson, métapsychologie freudienne, béhaviorisme, cognitivisme), le "pouvoir-être authentique " heideggérien lié à la question aristotélicienne du souci (le rapport de vérité que chacun

10 Paul Ricœur, Soi-même comme un autre, Paris, Éditions du Seuil, col. «L'ordre philosophique ", 1990; La Mémoire, l'histoire, l'oubli, Paris, Éditions du Seuil, coll. "L'ordre philosophique ", 2000; Parcours de la reconnaissance. Trois études, Paris, Gallimard, coll. «Folio/essais », 2007 [2004].

11 Barbara Cassin, L'Effet sophistique, Paris, Gallimard, 1995.

12 Ruth Amossy, La Présentation de soi : èthos et identité verbale, Paris, Presses universitaires de France, coll. "L'interrogation philosophique ", 2010. 
entretient avec lui-même), sans oublier le désir au sens de Lacan (ni pulsion au sens freudien, ni volonté de reconnaissance au sens hégélien), l'éthique de la discussion (Habermas), de la vertu (Wright, McIntyre), le déontologisme (Rawles)...

Cette énumération expéditive laisse entendre que la notion, le thème (le concept?) de l'identité fait grand florès tout au long de l'histoire de la philosophie. Comme le note à juste titre JeanClaude Kaufmann dans ses plus que "brefs prolégomènes philosophiques ", " [b]ien des questions d'aujourd'hui [sur l'identité] sont déjà posées [dans le cadre de l'histoire de la philosophie $]^{13}$ ". S'il ne s'agit pas de l'identité telle que formulée par la majorité des sciences humaines d'aujourd'hui ${ }^{14}$, il n'en demeure pas moins que nombre de «[...] développements [de l'histoire de la philosophie] peuvent s'appliquer à l'identité vue sous un angle plus existentiel ${ }^{15} "$.

Si le territoire ratissé par l'histoire de la philosophie est immense, que dire du discours de nos modernes sciences humaines et sociales sur le problème millénaire de la permanence et du changement? L'émergence, la popularité et la prospérité assez récentes du vocable identité dans le champ des sciences humaines datent de l'après Deuxième Guerre mondiale, notamment avec les travaux de Erik Homburger Erickson ${ }^{16}$, inspirés en partie par les recherches de Freud sur les identifications ${ }^{17}$. La gloire

13 Jean-Claude Kaufmann, op. cit., p. 16.

14 Ibid., p. 15. Que Kaufmann regroupe autour de «[...] l'interrogation, du point de vue de l'individu (ou du groupe), sur sa propre définition ("Quisuis-je? ») [...]».

15 Ibid., p. 16.

16 Erik Homburger Erickson, Childhood and Society, New York, WW Norton, 1963; Identity, Youth and Crisis, New York, WW Norton, 1968.

17 Tout en signalant ce que Freud doit à la philosophie, notamment aux identités de pensée et aux identités de perception, Kauffman fait de l'inventeur de la psychanalyse celui « [...] qui va introduire à la réflexion moderne sur l'identité "(Kauffman, op. cit., p. 25). Il cite à ce propos Laplanche et Pontalis : «le concept d'identification a pris progressivement dans l'œuvre de Freud la valeur centrale qui en fait, plus qu'un mécanisme psychologique parmi d'autres, l'opération par laquelle le sujet humain se constitue " (Jean Laplanche et Jean-Bertrand Pontalis, Vocabulaire de la psychanalyse, Paris, Presses universitaires de France, 1967, p. 188, cité dans Kauffman, op. cit., p. 25). Selon ce dernier, Freud insiste sur les dimensions "dynamique » et 
relativement récente de la notion d'identité, dont les débuts se situent aux alentours des années 1950-1960, est contemporaine d'une rupture radicale dans la fabrique du tissu social: «[...] l'émergence du sujet devenant (en théorie) maître de son futur. Le modèle du développement social est dorénavant celui de l'individu démocratique, libre de ses choix les plus divers (à chacun sa vérité, sa morale, son engagement dans des liens sociaux $)^{18}$ ». Selon Gilles Lipovetsky, les années 1950 inaugurent un "nouveau cycle historique des économies de consomma$\operatorname{tion}^{19}$ ", qui se traduit notamment par une croissance économique forte, une augmentation exponentielle de la productivité du travail, le développement fordien de l'économie à une échelle inédite et la multiplication du pouvoir d'achat des salaires par trois ou quatre. Cela aboutit à démocratiser « les rêves de l'Eldorado consumériste " de telle sorte que cette période de faste qui s'étend sur au moins trois autres décennies " [...] se présente comme le modèle pur de la "société de consommation de masse" 20 ». Il s'agirait là " [...] d'une immense mutation dont on ne soulignera jamais assez la radicalité instituante d'une rupture culturelle ${ }^{21}$ ". C'est dans cette conjoncture historique que les recherches sur l'identité commencent à mobiliser tout un pan des sciences humaines, comme si liberté et aisance économiques incitaient en parallèle un vaste questionnement sur soi.

Erikson vise à comprendre l'identité dans le cadre des relations entre l'individu, le groupe et la société. Ses objets de recherche de prédilection sont l'enfance (ses crises d'identité, ses phases de

" constructiviste " de l'identité; ses recherches laissent ainsi penser que l'identité, loin de renvoyer à un "donné préalable ", à une prise de position foncièrement substantialiste, rend possible la construction du sujet « [ ...] jour après jour par des identifications » (ibid., p. 25). À ce titre, le concept freudien d'identification " [...] permet de concevoir l'individu comme un processus, continuel et mouvant, ouvert sur son environnement social. [L'identification] est beaucoup plus qu'une simple imitation : une appropriation véritable. L'individu structure son moi par des échanges identificatoires avec ce qui l'entoure, en intériorisant des modèles et des images" (ibid., p. 25). Jean-Claude Kaufmann, op. cit., p. 20.

19 Gilles Lipovetsky, op. cit., p. 33.

20 Ibid., p. 33-34.

21 Ibid., p. 34. 
développement au regard de la vie humaine) et les indiens Lakota (leur rapport problématique à la modernisation de la société américaine). Ses analyses insistent sur la dimension processuelle à l'œuvre dans toute caractérisation identitaire; elles mettent en relief également les aspects subjectifs nécessaires à l'attribution de tout prédicat d'identification. Dynamique, complexe et ouverte sur la socialisation, cette double dimension de l'individuation moderne débouche sur une vaste quête identitaire qui accompagne, de phases en étapes, l'individu tout au long de son développement ${ }^{22}$. Cela dit, une autre conception de l'identité voit le jour dans les recherches d'Erickson, qui semble bel et bien remettre en question le concept d'identité comme processus subjectif ouvert sur le monde: l'identité enfin fixée au terme d'un parcours individuel singulier - comme si les diverses crises existentielles aboutissaient finalement à la constitution d'un sujet bien organisé émotionnellement et socialement, tout à fait conscient enfin de son identité personnelle, de sa permanence dans le temps. Cette ambivalence sera soulignée par plusieurs commentateurs $^{23}$ et, indépendamment des recherches de Erickson comme telles, elle permettra de caractériser au plus près les deux grandes options épistémologiques de la recherche sur l'identité des quelques cinquante dernières années: d'une part, une orientation essentialiste et, de l'autre, une vision plus volontiers existentialiste $^{24}$. Dans le sillage de cette ambivalence profonde, les recherches sur les relations triadiques individu-société-identité se multiplieront au fil des décennies dans le contexte de divers champs disciplinaires.

Le sociologue américain Robert King Merton élabore, pour sa part, une typologie des groupes de référence selon laquelle les identifications constituent des dispositifs indispensables à l'éla-

$22 \quad$ Erik Homburger Erickson, Identity..., op. cit.

23 Notamment Edmond-Marc Lipiansky, Isabelle Taboada-Léonetti et Ana Vasquez, "Introduction à la problématique de l'identité ", dans Carmel Camilleri et al. (dir.), Stratégies identitaires, Paris, Presses universitaires de France, 1990, p. 11; Jean-Claude Kaufmann, op. cit., p. 29.

24 Claude Dubar, La Crise des identités, Paris, Presses universitaires de France, 2000. Il en sera question plus bas. 
boration d'une définition de soi sur le plan individuel et collectif ${ }^{25}$. Ces dispositifs permettent à quiconque de s'identifier à des " individus de référence " ("role model»), des groupes ou des ensembles humains plus larges en fonction de critères qui définissent le comportement dans la perspective d'objectifs personnels ou culturels à réaliser. Or, comme les acteurs sociaux n'ont pas toujours la chance d'atteindre leurs objectifs, ils en viennent à une forme quelconque de déviance sociale par laquelle se creuse fatalement un écart entre l'individu et les normes collectives. Dans un autre contexte, Erving Goffman étudie les relations individus-société en mettant notamment l'accent sur les aspects symboliques en jeu, ainsi que sur les manières de se présenter et de se représenter soi-même dans une société démocratique qui exige un traitement de déférence des uns envers les autres ${ }^{26}$. Au centre de l'analyse goffmanienne de la modernité, une thèse forte qui souligne tant et tant la distance irréductible entre l'individu et la société, contrairement à une sociologie plus orthodoxe de type parsonien par exemple, selon laquelle l'individu en vient toujours à s'identifier à ses rôles sociaux. Chez Goffman, cette distance explique les difficultés liées aux interactions dans les sociétés démocratiques dites égalitaires. À vrai dire, la fragilité des situations sociales repose sur une double exigence: d'une part, celle de l'idéal d'être pleinement et entièrement soi vis-à-vis d'autrui et, de l'autre, celle de la déférence à lui témoigner en tout temps. L'épreuve des interactions sociales montre en fait que cette exigence à deux entrées constitue une sorte d'injonction contradictoire, sinon paradoxale, qui incite fortement l'individu à s'exprimer en adoptant des stratégies qui transgressent l'idéal d'égalité démocratique de sincérité et de déférence. Les analyses

$\overline{25}$ Robert King Merton, Social Theory and Social Structure, New York, Free Press, 1968.

26 Erving Goffman, La Présentation de soi. La mise en scène de la vie quotidienne I, trad. de l'anglais par Alain Accardo, Paris, Éditions de Minuit, coll. « Le sens commun », 1973 [1959]. 
que Goffman consacre à l'asile ${ }^{27}$ et au stigmate ${ }^{28}$ mettront en scène, de façon plus radicale encore, l'injonction paradoxale que constitue le principe de base de la sociabilité démocratique ("être soi en tout temps sans jamais pouvoir l'être »). Le caractère inhumain de l'asile consiste notamment en ceci que l'individu y est totalement dépossédé de son identité. On vise ainsi à en faire un patient dont la docilité voire la soumission physique et morale seront ses caractéristiques principales. On procède ensuite à une manière de resocialisation des patients au sein même du lieu asilaire, laquelle les incite souvent à commettre des actions illicites de tous ordres, en acte réfléchi de défiance envers l'autorité, mais surtout par refus catégorique du nouveau rôle qu'on leur assigne désormais. Cela montre sans équivoque que l'asile engendre son propre institutionnalisme maladif ${ }^{29}$. La question du stigmate sera aussi liée au cadre de l'idéal démocratique des échanges d'égal à égal. Comment en vient-on à établir une relation égalitaire avec quelqu'un de différent, avec " un individu que quelque chose disqualifie et empêche d'être pleinement reconnu par la sociétée ${ }^{30}$ "? Goffman montrera encore une fois à quel point l'idéal de sincérité et de déférence envers autrui (ici le stigmatisé) est mis à mal dans les interactions sociales. Il ne faut pas cependant perdre de vue que le sociologue considère l'individu stigmatisé en termes de relations sociales, non en fonction de ses attributs proprement dits. Aussi la distinction entre le stigmatisé et le normal relève-telle d'un processus social et d'une série de points de vue changeants selon les contextes (une personne dite normale dans un contexte pourra faire l'objet d'une stigmatisation dans un autre contexte). Ces points de vue peuvent en venir à établir et à définir des identités virtuelles et des identités sociales réelles, tant l'écart entre la caractérisation conférée à une personne stigmati-

$27 \quad$ Erving Goffman, Asiles. Études sur la condition sociale des malades mentaux, trad. de l'anglais par Liliane Lainé, Paris, Éditions de Minuit, coll. « Le sens commun », 1968 [1961].

28 Erving Goffman, Stigmate. Les Usages sociaux des handicaps, trad. de l'anglais par Alain Kihm, Paris, Éditions de Minuit, coll. «Le sens commun », 1975 [1963].

29 Erving Goffman, Asiles..., op. cit.

30 Eving Goffman, Stigmate..., op. cit., p. 7. 
sée et son identité réelle peut être plus ou moins grand. Dans tous les cas de figure, Goffman envisage cette problématique de l'écart dans le cadre de la double exigence démocratique soulignée plus haut; toute une gamme d'attitudes en découlent relativement à la difficulté morale à accepter le stigmate d'autrui, à tolérer une telle attitude chez autrui, ou bien chez soi-même, au rejet ou à l'acceptation hypocrite d'autrui en raison de son stigmate... Vis-à-vis de telles attitudes, et fortement conscient qu'elles fragilisent et rendent fausses les interactions, le stigmatisé met en pratique une véritable stratégie du faire semblant. Dans ce cadre, Goffman en viendra à définir le concept d'identité pour soi afin d'expliquer les sentiments de la personne stigmatisée, son comportement face à des gens dits normaux, à d'autres personnes stigmatisées ou à des professionnels qui tentent de lui venir en aide. Au terme de son analyse, Goffman signale notamment que l'individu stigmatisé devient vite un maître de la conscience réflexive de soi, mais malheureusement au détriment de son propre sentiment d'identité, sinon d'authenticité personnelle. En outre, il est aux prises avec des sentiments ambivalents puisqu'il recherche à la fois l'appartenance à son groupe, tout en nourrissant le désir de vivre sa vie comme il l'entend à l'écart de ce groupe. En somme, si le stigmatisé appartient à la société démocratique, il lui est également étranger. On comprend alors sous quel rapport la démocratie égalitaire, comme modèle d'intégration sociale, produit toute une panoplie de crises d'intégration et, par voie de conséquence, de crises d'identité.

Â Toulouse, un colloque important de sociologie en 1979 sur la thématique de la production et de l'affirmation identitaires insiste sur l'importance de l'identification et de la construction de l'identité, par opposition à la dyade identification-appartenance ${ }^{31}$. En politicologie, William James MacKenzie aborde la question de l'identité politique dans la perspective de pratiques discursives ${ }^{32}$.

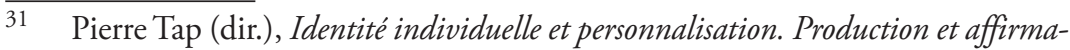
tion de l'identité, tome I et Identités collectives et changements sociaux, tome II, Toulouse, Privat, coll. "Sciences de l'homme », 1980. Sur cette question, on lira également Claude Dubar, La Crise..., op. cit., p. 8.

32 William James McKenzie, Political Identity, Harmondsworth, Penguin, 1978. 
Il souligne ainsi l'importance des récits mythiques, voire des narrations rituelles et, plus largement, du rôle fondamental assumé par le symbolisme dans l'organisation de toute identité politique. La fonction accordée aux productions symboliques de tous ordres le conduit également à insister sur l'aspect construit de l'identité. Dans son ouvrage L'Imaginaire national: réflexions sur l'origine et l'essor du nationalisme, Benedict Anderson met également en relief la fonction symbolique dans l'identification de l'individu à d'autres individus comme critère indispensable à la formation d'une conscience nationale ${ }^{33}$. À ses yeux, toute identité nationale s'avère une vaste construction socio-historique dont l'événement fondateur se perd dans la nuit des temps. Dans la monumentale Histoire de la vie privée en cinq tomes sous la direction de Philippe Ariès et Georges Duby, l'identification à un ou à des groupes sociaux, à des cultures nationales ou imaginaires, à des thématiques comme le travail, la religion, la famille..., s'exprime souvent par le truchement de pratiques discursives ou symboliques dûment reconnues, comme pour les $\mathrm{XIX}^{\mathrm{e}}$ et $\mathrm{XX}^{\mathrm{e}}$ siècles la presse, la radio, la télévision..., tant il est vrai que " [1]a vie privée n'est pas une réalité naturelle, donnée depuis l'origine des temps; c'est une réalité historique, construite de façon différente par des sociétés déterminées ${ }^{34}$ ".

Par ailleurs, qui dit identité dit aussi crise de l'identité, tant les identités modernes (mais pas seulement elles...) s'avèrent complexes, changeantes, hétérogènes, contradictoires et parfois incertaines, très souvent embrouillées par les discours qui visent à les ordonner en un ensemble relativement cohérent. Stuart Paul Hall prend acte du caractère polymorphe de ces manifestations identificatoires et remarque que, comme la notion d'identité n'a pas encore été dépassée dialectiquement, force est donc de

33 Benedict Richard O'Gorman Anderson, L'Imaginaire national : réflexions sur l'origine et l'essor du nationalisme, trad. de l'anglais par Pierre-Emmanuel Dauzat, Paris, Éditions La Découverte, coll. " Sciences humaines et sociales », 2006 [1983].

34 Antoine Prost, "Frontières et espaces du privé ", dans Histoire de la vie privée, tome 5, "De la Première Guerre mondiale à nos jours ", Philippe Ariès et Georges Duby (dir.), Paris, Éditions du Seuil, coll. " Points/histoire », 1999 [1985], p. 15. 
continuer à l'utiliser, de réfléchir à partir d'elle à tout un ensemble de phénomènes de la culture contemporaine qui supposent ou appellent un processus quelconque d'identification ${ }^{35}$. Il insiste aussi sur le fait que nous avons besoin d'une « [...] théorie de ce que sont les mécanismes par le biais desquels les individus comme sujets s'identifient [...] aux "positions" où ils sont convoqués; aux mécanismes par lesquels ils emménagent, produisent et donnent un style à ces positions ${ }^{36}$ ". Denis-Constant Martin signale que le sociologue Claude Dubar « [...] propose des éléments d'une telle théorie et illustre la nécessité de comprendre la crise multiforme des identités dans le monde actuel parce qu'il importe d'être mieux armé afin d'y apporter des solutions ${ }^{37}$ ". Dubar discerne dans les recherches sur l'identité deux grandes orientations: d'abord, l'orientation dite essentialiste, selon laquelle toute identité peut être réduite à une essence quelconque; dans ce cadre, la description de pratiques et l'énumération de traits communs permettraient de déterminer l'appartenance objective à un groupe; il serait du reste impossible de renier identité et appartenance au sens essentialiste sans faire fondre sur soi le qualificatif honteux de " traître "; ensuite, l'orientation dite nominaliste ou existentialiste, par laquelle Dubar jette le discrédit sur la soi-disant nature essentialiste de l'identité, tout en refusant «[...] la fatalité des appartenances et [en niant] l'existence des différences intrinsèques entre les être humains ${ }^{38}$ ". Dubar inscrit ses propres recherches dans cette seconde orientation, comme d'ailleurs la majorité des chercheurs en sciences humaines et sociales des dernières décennies.

D'autre part, les recherches qui situent la narrativité au centre des questionnements sur l'identité s'inscrivent également dans la

35 Stuart Paul Hall (dir.), Questions of Cultural Identity, London, Thousand Oaks, 1996, p. 1.

36 Ibid., p. 13-14. Traduction personnelle.

37 Denis-Constant Martin, "Écarts d'identité, comment dire l'autre en politique? ", dans Denis-Constant Martin (dir.), L'Identité en jeu : pouvoirs, identifications, mobilisations, Paris, Éditions Karthala, coll. " Recherches internationales ", 2010, p. 18.

38 Claude Dubar, La Crise..., op. cit., p. 2-4. Cité dans Denis-Constant Martin, "Écarts d'identité... ", loc. cit., p.18. 
seconde orientation définie par Dubar. À ce titre, l'identité trouve lieu et place à la jonction de l'intime et du public, de l'individuel et du collectif, de Soi et de l'Autre. Elle n'est pas sans se soumettre aux aléas et aux revers du temps humain, en somme aux conjonctures socio-historiques. Les travaux de Paul Ricœur ont montré que le Soi ne saurait exister sans l'Autre et, partant, l'individu sans la société, l'identité sans l'altérité. En outre, le Soi et l'Autre relèvent d'une dialectique serrée qui éprouve son régime de concordances et de discordances selon les coordonnées d'une puissante forme symbolique, le récit. Le récit rend donc compte de l'interaction sociale au sens propre; il enchevêtre trames narrative et discursive selon le jeu des points de vue antagonistes qui se donnent comme " [...] un mixte instable entre fabulation et expérience vive ${ }^{39}$ " au cœur d'une mise en intrigue de la vie et de l'expérience. La sociologue Margaret Somers, quant à elle, insiste sur le fait que la fabrication de récits, d'histoires et de mises en intrigue de la vie personnelle ou collective s'avère un aspect clef de la vie en société, car ces récits élaborent des relations intersubjectives auxquelles on peut se référer pour choisir nos allégeances idéologiques, nos lieux et personnes d'identification, nos groupes de référence ${ }^{40} \ldots$ Nombreux sont les historiens ${ }^{41}$, les anthropolo-

39 Paul Ricœur, Soi-même..., op. cit., p. 190-191.

40 Margaret Somers, "The Narrative Constitution of Identity: A Relational and Network Approach ", Theory and Society, vol. 23, no 5, 1994, p. 605-649.

41 Fernand Braudel, L'Identité de la France, tome I, "Espace et histoire ", Paris, Flammarion, coll. "Champs ", 1990 [1986]; Philippe Ariès, L'Homme devant la mort, Paris, Éditions du Seuil, coll. "Univers historique », 1977 et Le Temps de l'histoire, Paris, Éditions du Seuil, coll. «Univers historique ", 1986; Robert Muchembled, Culture populaire et culture des élites de la France moderne $\left(X V^{E}-X V I I I^{e}\right)$, Paris, Flammarion, coll. "Champs », 2002 [1978] et L'Invention de l'homme moderne. Culture et sensibilité en France du XV au XVIII e siècle, Paris, Fayard, coll. " Pluriel », 1994 [1988]. 
gues $^{42}$, les psychologues ${ }^{43}$, les politologues ${ }^{44}$, les philosophes ${ }^{45}$, les sociologues ${ }^{46}$ qui signalent l'importance de la dimension narrative dans la construction de l'identité personnelle ou collective. En guise d'exemple, le psychologue américain Jerome Bruner montre à quel point le récit est au principe de la construction de Soi. Le récit de Soi et, à plus forte raison peut-être, le récit élaboré sur Autrui permettent en effet à l'individu de se construire une identité et de l'affirmer dans ses rapports aux autres, à la faveur

$\overline{42}$ Marc Augé, Les Formes de l'oubli, Paris, Payot \& Rivages, coll. "Petite bibliothèque ", 2001; Pour une anthropologie des mondes contemporains, Paris, Aubier, 1994; Le sens des autres. Actualité de l'anthropologie, Paris, Fayard, 1994; "Culture et imaginaire : la question de l'identité ", Revue de l'Institut de sociologie, vol. 3-4, p. 51-61; Clifford Geertz, The Interpretation of Culture and Selected Essays, New York, Basic Books, 1973.

Jerome Bruner, Acts of Meaning, Cambridge (Mass.), Harvard University Press, 1990; Actual Minds, Possible Worlds, Cambridge (Mass.), Harvard University Press, 1986; Andrew Molly et al. (dir.), The Uses of Narrative: Exploration in Sociology, Psychology and Cultural Studies, New Brunswick, Transaction Publishers, 2004; Jean-Claude Filioux et al., Analyse d'un récit de vie : l'histoire d'Annabelle, Paris, Presses universitaires de France, 2005.

Jean-François Bayart, L'Illusion..., op. cit,; Jean-François Bayart et Jean-Pierre Warnier (dir.), Matière à politique, le pouvoir, le corps, les choses, Paris, Éditions Karthala, coll. "Recherches internationales ", 2004.

45 Alain Badiou, Le Siècle, Paris, Éditions du Seuil, coll. "L'ordre philosophique ", 2005; Jurgen Habermas, Connaissance et intérêt, trad. française par Gérard Clémençon, Paris, Gallimard, coll. "Tel », 1991 [1979]; Paul Ricœur, L'Idéologie et l'utopie, Paris, Éditions du Seuil, coll. " Points/essais ", 1997; La Mémoire..., op. cit.,; Parcours..., op. cit.; Michel Meyer, Le Philosophe et les passions. Esquisse d'une histoire de la nature humaine, Paris, Presses universitaires de France, coll. "Quadrige ", 2007; Charles Taylor, Les Sources du moi. La formation de l'identité moderne, trad. française par Charlotte Melançon, Montréal, Boréal, coll. "Compact », 2003 [1992], Charles Taylor et Ami Gutman, Multiculturalisme: différence et démocratie, Paris, Flammarion, coll. "Champs », 1997.

46 Anthony Giddens, Modernity and Self-Identity. Self and Society in the Late Modern Age, Cambridge, Polity Press, 1991; Claude Dubar, La Crise..., op. cit.; Jean-Claude Kaufmann, LInvention de soi..., op. cit.; François de Singly, Les Uns avec les autres: quand l'individualisme crée du lien, Paris, Armand Colin, coll. "Individu et société ", 2003; Libres ensemble. L'Individualisme dans la vie commune, Paris, Nathan, coll. "Essais et recherches ", 2000; avec Daniel Bertaux (dir.), Le Récit de vie, Paris, Armand Colin, " coll. "L'enquête et ses méthodes ", 2005; Alain Touraine, Un Nouveau paradigme. Pour comprendre le monde d'aujourd'hui, Paris, Fayard, coll. " Biblio/essais ", 2008 [2006]. 
de nombreuses catégories, aussi diverses les unes que les autres, bien que souvent interdépendantes, comme la découverte, l'exploration des sentiments, le deuil, la dépression et le conflit ${ }^{47}$. Par la narrativité et la présence dessinée en creux de l'Autre, le sujet prend conscience du caractère à géométrie variable de son identité. Aussi devient-il de plus en plus conscient de lui-même et des autres par le biais de la réflexivité, laquelle implique des ajustements mineurs ou des transformations spectaculaires de soi selon le cas.

En outre, l'identité construite à travers une narration de Soi où l'Autre s'affirme à tout moment permet de comprendre à quel point Soi et Autre s'entremêlent si profondément. Mieux peutêtre que quiconque, Paul Ricour a su rendre compte de cette dialectique quasi inextricable:

[...] l'ipséité du soi-même implique l'altérité à un degré si intime que l'un ne se laisse pas penser sans l'autre, comme on dirait en langage hégélien. Au "comme" [du titre: Soi-même comme un autre], nous voudrions attacher la signification forte non pas seulement d'une comparaison - soi-même semblable à un autre -, mais bien d'une implication: soi-même en tant que... autre ${ }^{48}$.

Autrement dit, le Soi dépend intimement de la catégorie de l'Autre, non seulement quant aux états antérieurs de soi-même (ce qu'il était jadis), mais encore au regard de ce qu'il est hinc et nunc, puis de ce qu'il adviendra de lui dans un futur encore incertain... Dans cet ordre d'idées, la compréhension de Soi passe par toute une série de rapports à l'Autre; elle implique des transformations, des évolutions, des négociations de soi à soi indispensables à l'affirmation, à la consolidation ou à l'épanouissement de Soi.

Ce parcours quelque peu sommaire permet d'en arriver à certaines conclusions qui portent à conséquence pour toute analyse de l'identité individuelle ou collective. D'abord, l'identité ne saurait être considérée comme une essence, une essence dont les traits regroupés en noyau fixe s'opposeraient à la dialectique de la permanence et du changement évoquée plus haut. Si tel était

$47 \quad$ Jerome Bruner, Acts of Meaning..., op. cit.

48 Paul Ricœur, Soi-même..., op. cit., p. 14. 
le cas, l'identité ainsi conçue jetterait un immense défi à la temporalité humaine dans ses nombreuses dimensions, à Soi et à l'Autre comme noud inextricable de relations - peut-être y perdrait-on la fabrique de l'humain en faisant l'impasse sur la temporalité au sens large. En insistant sur les aspects contextuels de l'identité comme construction et comme processus, les sciences humaines et sociales ont mis en relief son caractère à la fois relationnel et dialectique. Loin d'être une substance intemporelle, l'identité renvoie à une permanence dans le temps incessamment soumise à l'épreuve du changement. En un mot, les identités n'ont pas d'existence intrinsèque, mais comme le souligne si bien Martin " [...] les usages qui en sont faits et leurs effets sont bien réels, et ont été souvent dramatiques ${ }^{49}$ ». D’autre part, le caractère inextricable des rapports entre Soi et Autre, qui implique que tout sujet peut se représenter à soi-même tout en se remémorant les étapes, les phases ou les événements marquants qui l'ont fait advenir comme sujet de parole et d'action en face d'Autrui, atteste du caractère à la fois multidimensionnel et variable de l'identité. La permanence et le changement dans le cadre de la dialectique de Soi et de l'Autre trouvent leurs modalités de signification dans la dimension narrative de toute expérience humaine véritablement réflexive. À ce titre, la régulation de l'identité par le récit (de soi) constitue une forme de médiation et de mémoire dont la fonction centrale consiste à consolider ou à renouveler l'individu et le groupe dans la fixation de leurs croyances sous la topique générale de la permanence et du changement. Du reste, on oublie souvent de signaler que les représentations à teneur identitaire insistent davantage sur le pôle de la permanence. En cela, elles comportent une visée d'idéalisation de la réalité qui peut aller jusqu'à faire l'impasse sur les dimensions associées au pôle du changement.

\section{Francisation et mesures disciplinaires}

En quoi cette synthèse partielle des travaux réalisés en sciences humaines et sociales informe-t-elle l'analyse de l'identitaire

49 Denis-Constant Martin, "Écarts d'identité... ", loc. cit., p. 24. 
franco-manitobain dans notre corpus de référence? Tout d'abord, on ne saurait inscrire les diverses formulations de cet identitaire au chapitre d'un quelconque essentialisme vitaliste ou théorique. Si tel était le cas, on en viendrait à ignorer le caractère hautement relationnel et dialectique de toute construction identitaire. Ensuite, on reléguerait aux calendes grecques la complexité relative aux modes d'imbrication de Soi et de l'Autre, tant au plan des relations humaines que de leurs représentations discursives. Cela reviendrait à ignorer la trame narrative propre à la socialisation humaine. C'est pourquoi nous inscrivons notre analyse dans l'orientation existentialiste telle que définie par Dubar $^{50}$, tout en insistant sur la dimension narrative de l'expérience et des médiations sociales: mise en intrigue de Soi et de l'Autre comme aspect essentiel de la vie en société.

Nombre de publi-reportages du corpus de référence abordent de manière implicite ou explicite la question Soi et de l'Autre en milieu scolaire. Au centre de cette thématique à ramifications multiples, l'incontournable problème de la francisation, son rôle et son importance pour contrer les risques d'assimilation: «En milieu minoritaire, nous sommes tous en francisation $[\ldots]^{51}$ ", remarque Monique Courcelles (la coordonnatrice du programme de francisation à la Division scolaire franco-manitobaine. "Quand on se rend compte de cette réalité, on change un peu la façon de faire les choses dans nos écoles ${ }^{52}$. " On verra que cette « façon de faire les choses » met en jeu les deux logiques complémentaires formulées dans le cadre de notre hypothèse de travail: 1) une logique de l'action rationnelle et de la créativité personnelle qui renvoie à une problématique générale de la réflexivité; 2) une logique dialectique de Soi et de l'Autre (le monde anglophone), implicite certes, mais non moins réelle et instituante, car elle incite aussi bien à la réflexion qu'à l'action. D'abord, on signale que " [...] l'apprentissage de la langue passe

\footnotetext{
$\overline{50}$ Claude Dubar, La Crise des identités..., op. cit., p. 2-4.

51 Daniel Bahuaud, «La Francisation en évolution ", La Liberté, vol. 95, n²4, 2008, p. 21.

52 Ibid., p. 21.
} 
par le vécu des étudiants $[\ldots]^{53}$ " et que cet apprentissage augmente leurs chances de réussite s'il se réalise " [...] à partir du concret de la vie de tous les jours [plutôt que] dans l'isolement ${ }^{54}$ ». Les jeux collectifs servent cet apprentissage, car « le jeune acquiert son vocabulaire en jouant et en travaillant avec les autres ${ }^{55}$ ». On invite ainsi les plus francisés à apporter leur aide à ceux qui le sont moins, ou pas. Si l'on encourage si fortement la participation estudiantine, on sollicite également les parents «[...] à réfléchir sur la place qu'ils accordent à la langue et à la culture françaises, en classe, à la maison, dans leur vie personnelle ${ }^{56}$ ". Dès lors, une série de questions se laissent formuler qui engagent la responsabilité de tous sous l'angle de l'action rationnelle et de la créativité personnelle: la télévision, en anglais ou en français? La bibliothèque familiale, davantage de livres anglophones ou francophones? Le même questionnement est reconduit sur les autres industries culturelles: radio, cinéma, Internet, réseaux sociaux, etc.

Aussi bien dire que les articles qui font référence à la francisation insistent sur l'importance d'une prise de conscience générale, afin que " $[. .$.$] le français soit une chose vécue chez soi { }^{57}$ ", comme d'ailleurs dans l'environnement scolaire (la salle de classe, les couloirs, les aires de jeu... $)^{58}$. Diverses mesures sont ainsi prises et elles incitent toutes sans exception à l'action rationnelle et à la création personnelle à l'école. Par exemple, les élèves les plus anglicisés ou les plus anglophones ne sont « [...] pas retirés de la salle de classe pour recevoir une formation plus intensive ${ }^{59}$ ". Ils recevront leur francisation par " [...] le biais d'exercices et d'activités auxquels toute la classe $[\ldots]^{60}$ " est conviée. On croit

53 Ibid., p. 21.

54 Ibid., p. 21. Le " concret de la vie de tous les jours ", c'est-à-dire, du moins en partie, je me permets d'y insister encore une fois : l'omniprésence massive du fait anglophone.

55 Ibid., p. 21.

56 Ibid., p. 21.

57 Ibid., p. 21.

58 Ibid., p. 21.

59 Ibid., p. 21.

60 Ibid., p. 21. 
que cette approche dite inclusive de la pédagogie n'étiquette personne et permet de créer des conditions optimales d'apprentissage et d'intégration sociale. Dans cette optique, les plus francisés participent au processus global d'apprentissage. S'il en est ainsi, on conçoit aisément que les meilleurs élèves doivent faire appel à toutes les ressources de leur créativité pour venir en aide aux élèves moins doués. Les uns et les autres acquièrent du coup une marge de manœuvre qui favorise l'autoréflexivité et les relations intersubjectives dans le cadre de socialisation que constitue l'école.

En outre, la francisation la plus intense serait « [...] de la maternelle à la deuxième année, [mais elle demeure] un processus qui dure pendant tout le cheminement scolaire ${ }^{61} "$. Les écoles Aurèle-Lemoine et Précieux-Sang considèrent l'approche pédagogique inclusive si importante qu'elles vont même jusqu'à tenir des séances d'information et de formation à l'intention des « [... enseignants, $[\mathrm{du}]$ personnel de soutien y compris les concierges, [des] parents et [des] élèves du niveau secondaire $[. . .]^{62}$ " et ce, en poursuivant l'objectif suivant: que tous « [...] puissent faire l'inventaire de leur identité et [de] leur engagement francophone $^{63}$ ". Le directeur de l'école communautaire Aurèle-Lemoine, Serge Carrière, explique en ces termes cette problématique identitaire francophone:

C'est très important de bien comprendre la réalité d'être francophone dans un milieu minoritaire [...] Ces sessions l'expliquent et nous le rappelle. Elles soulignent l'importance de l'école, et du foyer et de la communauté en général dans la réalisation de notre mission. Ce n'est pas juste une poignée d'enseignants du niveau primaire qui doivent assumer la responsabilité du dossier. C'est la responsabilité de tout le monde (ibid., p. 21).

On ne saurait en douter à la lumière de ces propos: la responsabilité est conçue sous le rapport d'une action rationnelle menée en tout temps, d'une créativité personnelle et collective qui exige une implication soutenue, des ressources cognitives non négli-

\begin{tabular}{ll}
\hline 61 & Ibid., p. 21. \\
62 & Ibid., p. 21. \\
63 & Ibid., p. 21.
\end{tabular}


geables mobilisées à la suite d'une prise de conscience généralisée ou réflexivité. Ce sont là les conditions qui permettent de faire front commun contre le fait anglophone. Sans jamais évoquer une forme quelconque d'assimilation, fût-ce seulement au plan linguistique, on préfère parler de l'" inventaire de l'identité francophone " et de mesurer l'" engagement " de chacun à la cause francophone de la communauté. Deux points importants doivent ici être soulignés: d'abord, aucune définition comme telle de l'identité franco-manitobaine n'est proposée; ensuite, la problématique de l'identité apparaît dans un contexte implicite d'opposition au monde anglophone, ne serait-ce que parce que l'engagement à une cause implique le fait que celle-ci puisse connaître un revers de fortune. La question identitaire suppose donc plus une manière d'être (résistance, combat, engagement inlassablement reconduit...) qu'une définition en compréhension. À ce titre, la présence anglophone engage bien plus à l'action qu'elle impulse une véritable définition de Soi par l'exclusive.

Par ailleurs, les nombreux publi-reportages du corpus sur la rentrée scolaire mettent aussi en scène les logiques de l'action rationnelle et de la créativité personnelle. Temps fort de l'année scolaire, la rentrée constitue le moment tout désigné pour énoncer l'un des mandats des écoles de la Division scolaire franco-manitobaine: la francisation. Par exemple, l'article intitulé "Bien fêter la rentrée ${ }^{64}$ » signale que l'École élémentaire NotreDame-de-Lourdes a fêté la rentrée sous le thème: "Je crée du nouveau chaque jour ». Les élèves ont créé des affiches " [...] en se laissant inspirer par leur imagination ${ }^{65} »$. Plus tard, ces affiches ont été mises en valeur lors d'une célébration eucharistique qui insistait «[...] sur l'importance de développer les dons et les talents qui nous sont donnés ${ }^{66} "$, comme le souligne la directrice de l'école, Jocelyne LeBlanc Boulet. Des jeux coopératifs ont eu lieu en fin de journée et les équipes gagnantes se sont méritées des prix. Un autre article portant sur la rentrée scolaire, intitulé $\overline{64}$ Daniel Bahuaud, «Bien fêter le rentrée ", La Liberté, vol. 95, nº 25, 2008, p. 21.

65 Ibid., p. 21.

66 Ibid., p. 21. 
celui-ci «Un accueil chaleureux ${ }^{67}$ ", signale avec emphase que la Division scolaire franco-manitobaine forme une "grande famille " et que l'école Saint-Agathe insiste sur la créativité de ses aînés pour présenter leur nouvelle famille scolaire aux plus jeunes. Les activités de la journée prévoient la formation de huit groupes d'élèves afin de créer une " ambiance familiale ». À l'École Taché, le thème de la rentrée scolaire de 2008 s'énonçait comme suit: "Construisons et célébrons ". Les professeurs étaient habillés " comme des ouvriers qui devaient construire une maison ${ }^{68}$ ". Le publi-reportage signale à quel point l'ambiance était à la fois " festive " et " comique ", rythmée de chants en swahili accompagnés de djembés africains. Les professeurs ont joué une pièce de théâtre intitulée "Construisons et célébrons ". La directrice de l'école signale que ces derniers à eux seuls ne peuvent pas construire un édifice, mais que les résultats seraient bien meilleurs si tout le monde mettait l'épaule à la roue. Au-delà du caractère moral de cette fable, encore une fois action rationnelle et créativité de tous les élèves sont sollicitées, notamment dans l'interprétation de la saynète théâtrale. À l'école Noël-Richtot, la journée d'accueil «[...] était axée sur le respect des autres, le parler français et la santé, tant émotionnelle que physique ${ }^{69}$ ". Les élèves pouvaient « [...] visiter huit stations sur les thèmes de la journée $^{70}$ ". Les responsables de la station ayant pour thème "le respect des autres " n'abordaient pas la question de la punition en cas d'infraction mineure ou majeure, mais invitaient plutôt les élèves à réfléchir sur le "comportement positif " à adopter. On signale tout simplement qu'ils ont formulé de très bonnes idées sur le respect et qu'ils ont fait des dessins pour les illustrer. Action rationnelle, création personnelle et autoréflexivité entrent aussi en jeu dans ces exemples.

"Fêter, créer, gagner des prix, construire ensemble, respecter autrui, parler français dans une ambiance familiale ": autant

\footnotetext{
67 Daniel Bahuaud, "Un Acceuil chaleureux », La Liberté, vol. 95, n 27, 2008, p. 27.

68 Ibid., p. 27.

69 Ibid., p. 27.

$70 \quad$ Ibid., p. 27.
} 
d'aspects positifs d'un récit de Soi exemplaire axé sur le renforcement identitaire et le libre-arbitre. Le travail collectif, l'entraide et le soutien mutuel montrent bien qu'il ne s'agit pas de combattre individuellement les risques d'assimilation par la culture anglophone omniprésente, mais de le faire collectivement dans une " ambiance familiale ", à la maison comme à l'école. Tout cela, dans le cadre d'activités rationnelles et créatrices tant sur le plan personnel que collectif. Encore une fois, il ne s'agit pas de dire noir sur blanc ce que serait l'identité franco-manitobaine, mais d'insister implicitement sur la résistance, le combat, l'action qu'implique l'engagement à une cause. Et pas seulement au cours des années de scolarisation, mais tout au long d'une vie. À preuve, la formation des parents, du personnel de soutien des écoles... Encore une fois, l'Autre dessine sa présence sur le mode de... l'absence.

D'autre part, la thématique de la discipline en milieu scolaire est omniprésente dans le corpus de référence. Plusieurs publireportages portent en effet sur cette thématique; on rencontre aussi des remarques à ce sujet dispersées à travers le corpus de référence. Thèmes et remarques ponctuelles insistent tous sur l'importance de "responsabiliser " les élèves, non de les punir, sur la nécessité d'une prise de conscience générale, non sur le motif de la peur. On veut ainsi en finir avec une pratique disciplinaire fondée sur l'échec, le reniement de soi et la punition honteuse. C'est dans ce contexte que la problématique de l'action rationnelle, de la créativité personnelle et de l'autoréflexivité comme traits essentiels du récit de Soi exemplaire entrent encore une fois en ligne de compte. En effet, il s'agit de mettre l'accent sur la responsabilité des élèves, mais de le faire de manière positive et enrichissante. Dans un publi-reportage intitulé « La discipline sans contraintes ", on explique " [...] une approche innovatrice au défi de la discipline ${ }^{71}$ ». L'école régionale Saint-Jean-Baptiste croit en fait avoir trouvé une solution à l'indiscipline dans une approche qu’on nomme la réparation. Edmée Sabourin, la

\footnotetext{
71 Daniel Bahuaud, « La Discipline sans contrainte », La Liberté, vol. 95, nº 22, 2008, p. 23.
} 
directrice de cette école, explique que cette approche vise à responsabiliser les élèves en remettant « [...] entre [leurs] mains la responsabilité de [leurs] actes, dont [ils doivent toujours] assumer les conséquences ${ }^{72}$ ». Aussi encourage-t-on fortement les élèves à "réparer les gestes [d'indiscipline] commis ${ }^{73}$ ". Ils doivent dès lors faire preuve de raison et de création dans la recherche d'une solution; et celle-ci " [...] doit être valorisante pour tous [...] $]^{74}$ " afin que la solution proposée puisse prendre valeur de norme pour le groupe. La directrice signale que « [c] ela demande de la réflexion et un certain effort ${ }^{75} »$; elle poursuit en disant qu'il est nécessaire que « [...] la solution vienne de l'enfant, au lieu de voir les adultes diriger sa discipline ${ }^{76} »$. On vise ainsi à rendre l'élève plus autonome sur le plan moral, et surtout plus respectueux de ses condisciples et maîtres. L'école Saint-Jean-Baptiste a donc « [...] revu comment elle s'y prend pour faire de la discipline $^{77}$ " et cette réforme concerne tout un chacun, car " [l]es élèves, les enseignants, le personnel de soutien et l'administration ont décidé ensemble quel sera le climat scolaire ${ }^{78} »$. Les élèves ont formulé des règlements positifs et ils ont aussi élaboré une manière de contrat social. Selon la directrice, il s'agit là d'une preuve « [...] [qu'ils] sont prêts à faire respecter les consignes qu'ils ont euxmêmes établies ${ }^{79}$ ".

L'exemple disciplinaire est intéressant parce qu'il permet de comprendre que les logiques de l'action rationnelle et de la création personnelle et collective renvoient non seulement à un cadre éthique de la formation de l'individu, mais encore à un cadre identitaire d'action nécessaire à l'engagement francomanitobain. Cela revient à dire que les logiques à l'œuvre au niveau de la francisation (action rationnelle, créativité personnelle et collective, réflexivité) se retrouvent également dans la problé-

$\begin{array}{ll}72 & \text { Ibid., p. } 23 . \\ 73 & \text { Ibid., p. } 23 . \\ 74 & \text { Ibid., p. 23. } \\ 75 & \text { Ibid., p. 23. } \\ 76 & \text { Ibid., p. 23. } \\ 77 & \text { Ibid., p. 23. } \\ 78 & \text { Ibid., p. 23. } \\ 79 & \text { Ibid., p. 23. }\end{array}$


matique disciplinaire. Les logiques du récit de Soi s'énoncent ainsi sur deux plans comportementaux distincts mais interdépendants; ce qui les renforce sans doute. Quoi qu'il en soit, un principe de continuité apparaît au cour du récit de Soi exemplaire dont les deux trames logiques mettent en évidence un même devoir-être vis-à-vis de réalités différentes.

\section{Les titres des publi-reportages: une pratique de l'éloge et de l'engagement}

Les titres des publi-reportages à épisodes biographiques louangent hyperboliquement les réalisations des élèves de la Division scolaire franco-manitobaine. À cet égard, les titres débordent leur fonction désignative et relèvent d'une véritable rhétorique de l'éloge. Quelques exemples de ces titres, parmi tant d'autres: "Bravo Charles ${ }^{80 !}$ ", " L'excellence à l'honneur ${ }^{81}$ ", "Un talent démasqué $^{82}$ ", "L'étoffe des héros ${ }^{83}$ ", "Un esprit de gagnant ${ }^{84}$ ", "Bravo les championnes ${ }^{85}$ "... Comme on le remarque, de tels titres comportent une dimension iconique évidente, qui caractérise l'élève dont il est question. Par son titre, le récit de Soi isole très souvent une image à caractère hyperbolique et élogieux. Comme de nombreux chercheurs en sémiotique ${ }^{86}$, en communication ${ }^{87}$ et en sociologie ${ }^{88}$ l'ont signalé, l'image joue un rôle non

80 Daniel Bahuaud, " Bravo Charles! ", La Liberté, vol. 92, n 39, 2006, p. 22.

81 Daniel Bahuaud, "L'Excellence à l'honneur ", La Liberté, vol. 92, n 39, p. 23.

82 Daniel Bahuaud, "Un Talent démasqué ", La Liberté, vol. 92, n 40, 2006, p. 25.

83 Daniel Bahuaud, «L'Étoffe des héros », La Liberté, vol. 92, n 43, 2006, p. 17.

84 Daniel Bahuaud, "Un Esprit de gagnant ", La Liberté, vol. 92, n 45, p. 24.

85 Daniel Bahuaud, "Bravo les championnes ", La Liberté, vol. 92, n 48, p. 22.

86 Guy Lochard et André Boyer, La Communication médiatique, Paris, Éditions du Seuil, coll. "Mémo ", 1998; Jean-Pierre Ezquenazy, L'Écriture de l'actualité. Pour une sociologie du discours médiatique, Grenoble, Presses universitaires de Grenoble, 2002; Yves Lavoinne, Le Langage des médias, Grenoble, Presses universitaires de Grenoble, 1999.

87 Lucien Sfez, Critique de la communication, nouvelle édition entièrement refondue et augmentée, Paris, Éditions du Seuil, coll. "Point ", 1992; Philippe Breton, La Parole manipulée, Paris, Éditions La Découverte, coll. «Poche/essais ", 2000 [1997].

88 Anne Sauvageot, Voirs et savoirs. Esquisse d'une sociologie du regard, Paris, Presses universitaires de France, coll. "Sociologie d'aujourd'hui ", 1994; 
négligeable dans le cadre de tout processus identitaire. Certains avancent même que les images sont des « [...] éléments concrets d'élaboration identitaire, guidant à chaque instant les positionnements dans les interactions ${ }^{89} "$, les rapports intersubjectifs ou sociaux. Ces images seraient donc " [...] bien plus centrale[s] qu'on ne croit $[\ldots]^{90}$ " à première vue; elles seraient en fait un élément incontournable pour la construction sociale de l'indi$v \mathrm{du}^{91}$. Du reste, ces images comportent toutes une forte charge émotive.

Encore faut-il bien comprendre le contexte d'élaboration de ces images dans le corpus de référence, car elles peuvent tendre des pièges à l'interprétation. Ces images prennent leur sens dans un cadre de signification bien circonscrit. Elles renvoient à la communauté franco-manitobaine une représentation des activités qui ont eu lieu au sein de sa division scolaire ; elles sont toutes proposées par le signataire des publi-reportages, c'est-à-dire très rarement élaborées par les adolescents eux-mêmes. Si elles ne visent pas à cerner ces derniers dans la complexité de leurs coordonnées identitaires empiriques, elles ne relèvent pas pour autant de la superficialité des apparences. Certes, ces images apparaissent schématiques, réductrices et incapables d'expliquer la complexité d'une véritable subjectivité à l'œuvre. Mais là n'est pas leur raison d'être, tant s'en faut, ni sur le plan du publireportage, ni sur le plan social. La fonction de ces images dans le discours consiste à construire un lieu de représentation; on réalise alors quelles constituent un commentaire mélioratif sur la logique de l'action rationnelle, de la créativité personnelle et de l'autoréflexivité des élèves.

Anne-Marie Constalat-Founeau, Identité sociale et dynamique représentationnelle, Rennes, Presses universitaires de Rennes, coll. "Didactique et psychologie sociale ", 1997; Jan Stets et Peter Burke, "A sociological Approach to Self and Identity ", dans Marc R. Leary et June Price Tangney (dir.), Handbook of Self and Identity, New York, Gilfred Press, 2002; Jean-Claude Kaufmann, L'Invention de soi..., op. cit.

89 Jean-Claude Kaufmann, L'Invention de soi..., op. cit., p. 69.

90 Ibid., p. 69.

91 Anne-Marie Sauvageot, Voirs et savoirs..., op. cit., p. 38. 
La fonction informative du titre est donc subordonnée à une fonction indicielle plus générale qui participe d'une stratégie de l'éloge. Autrement dit, les titres prennent une valeur d'illustration. Un tel procédé relève de l'argumentation par induction. Comme on le sait, ce mode de raisonnement tend à la généralisation à partir de cas particuliers; les formes les plus usitées de l'induction dans le corpus de référence sont l'exemple, l'illustration et le modèle. Ce qu'il y a d'intéressant dans le procédé de l'illustration, c'est qu'il tend à renforcer une thèse considérée comme admise, en lui donnant une apparence vivante et concrète. Lillustration vise donc moins à convaincre qu'à frapper vivement l'imagination. C'est d'ailleurs pourquoi son efficacité ne dépend pas nécessairement d'un acte de référence véridicte. Si l'on prend en considération la nature hyperbolique des titres dans le corpus de référence, on réalise que ces derniers orientent fortement la communication sur le plan émotif. Le titre hyperbolique témoigne ainsi du degré d'engagement du signataire des publi-reportages et de la manière dont celui-ci s'inscrit dans des attitudes attendues ou admises. C'est tellement vrai qu'on ne saurait titrer un publireportage en adoptant un point de vue critique à l'égard du récit de Soi d'un adolescent de la Division scolaire franco-manitobaine. Le degré d'engagement de l'auteur des publi-reportages doit donc être compris, entre autres positionnements vis-à-vis de la communauté, à la lumière des titres élogieux choisis.

En outre, cette rhétorique de l'éloge est créatrice de valeurs axiologiques spécifiques comme l'héroïsme, l'excellence, le dépassement de soi... Elle renvoie à un univers d'actions et de prestations hors du commun. Comme tel, le titre serait déjà une première orientation de la signification au profit d'une axiologie positive qui relève d'une attitude attendue. Cela permet de comprendre que les titres du corpus de référence intègrent une dimension sociale indéniable où l'Autre se profile toujours en arrière-plan. À vrai dire, les images et les valeurs axiologiques que les titres véhiculent permettent une double ouverture à la socialisation (ou à la formulation du social du point de vue identitaire): d'une part, ils inscrivent au sein d'un univers de 
signification la marque d'un collectif, d'un Nous identitaire, sur la base d'un degré d'engagement maximal de l'auteur des publireportages et du mandat du journal La Liberté; d'autre part, en leur qualité de commentaire mélioratif, ils induisent des interprétations de type: "Notre jeunesse est véritablement digne de mention " - signe indubitable s'il en est que l'engagement identitaire porte fruit. Les titres concrétisent les valeurs liées aux savoir et au devoir-être identitaires; ils prennent le sens d'une sanction mass-médiatique laudative et témoignent d'un engagement personnel maximal.

\section{Conclusion}

Le récit de Soi occupe une place de prédilection dans les publireportages du corpus de référence. Les élèves du primaire tout comme du secondaire, les directeurs et les directrices d'école de la Division scolaire franco-manitobaine en sont les énonciateurs principaux, mais tout intervenant (responsables des programmes éducatifs, maîtres ou employés de soutien, parents ou tuteurs) qui prend la parole au nom de la communauté contribue à l'élaboration du récit de Soi franco-manitobain. Ce récit propose des thématiques, des épisodes de vie et des scénarios intertextuels analogues les uns aux autres à l'échelle du corpus, qui insistent tant et tant sur les motifs du combat, de la lutte, de la résistance, de l'entraide et de la famille afin de faire front commun contre la menace de l'assimilation linguistique et culturelle. Cet argumentaire exploite de manière simple et concrète le savoir-être et le devoir-être identitaires dans la perspective de la responsabilité individuelle et collective, voire de l'engagement de toute une vie.

Les exemples de francisation, de discipline et de rentrée scolaire déploient la logique de l'action rationnelle, de la créativité personnelle et de l'autoréflexivité. De tels ressorts discursifs se traduisent dans le monde de l'action pratique et rendent ainsi possible une administration de la question identitaire selon des critères d'utilité et de rendement communautaires. Le savoir-être et le devoir-être identitaires relèvent par conséquent de l'ordre public; ils sont pris en charge par des paroles, des comportements, 
des programmes, des manières de faire qui visent non seulement la formation de l'élève, mais aussi celle des enseignants, des employés de soutien et des parents dans le cadre élargi de l'école comme " unité familiale ». Â ce titre, ils renvoient à un répertoire de croyances, de valeurs et de normes comportementaux axés sur l'engagement de chacun au profit de tous. Savoir-être et devoirêtre identitaires apparaissent donc comme des enjeux non négligeables dans les publi-reportages.

En véhiculant ces enjeux, le récit de Soi rencontre immanquablement l'Autre sur son passage, le fait anglophone. Si celui-ci incarne une présence implicite, il n'en représente pas moins ce par qui ou par quoi il importe de se définir. Or le savoir-être et le devoir-être identitaires ne prennent pas dans les publireportages la forme homogène d'une identité définie comme telle, dont les valeurs essentielles seraient un héritage historique, une religion ou une appartenance politique quelconque. Le récit de Soi est bien davantage une manière de réagir face aux difficultés de maintenir vivantes une langue et une culture. Cela revient à dire que le récit de Soi exemplaire est traversé par une composante anglophone que l'on découvre en arrière-plan. Le récit de Soi se conjugue au pluriel; c'est le récit d'un collectif d'adhésion traversé de part en part par l'Autre de la culture franco-manitobaine. 


\section{Bibliographie}

Amossy, Ruth, La Présentation de soi: èthos et identité verbale, Paris, Presses universitaires de France, coll. «L'interrogation philosophique », 2010, 280 p.

Anderson, Benedict Richard O'Gorman, L'Imaginaire national. Réflexions sur l'origine et l'essor du nationalisme, trad. de l'anglais par PierreEmmanuel Dauzat, Paris, Éditions la Découverte, coll. "Sciences humaines et sociales ", 2006 [1983], 224 p.

Ariès, Philippe, L'Homme devant la mort, Paris, Éditions du Seuil, coll. "Univers historique », 1977, $641 \mathrm{p}$.

Ariès, Philippe, Le Temps de l'histoire, Paris, Éditions du Seuil, coll. "Univers historique ", 1986, 255 p.

Ariès, Philippe et Georges Duby (dir.), Histoire de la vie privée, "De la Première Guerre mondiale à nos jours ", tome V, Paris, Éditions du Seuil, coll. « Points/histoire », 1999 [1985], 625 p.

Augé, Marc, "Culture et imaginaire: la question de l'identité », Revue de l'Institut de sociologie, vol. 3-4, 1988, p. 51-61.

Augé, Marc, Les Formes de l'oubli, Paris, Payot \& Rivages, coll. "Petite bibliothèque ", 2001, 121 p.

Augé, Marc, Pour une anthropologie des mondes contemporains, Paris, Aubier, $1994,195 \mathrm{p}$.

Augé, Marc, Le Sens des autres. Actualité de l'anthropologie, Paris, Fayard, 1994, $199 \mathrm{p}$.

Badiou, Alain, Le Siècle, Paris, Éditions du Seuil, coll. "L'ordre philosophique ", 2005, $255 \mathrm{p}$.

Bahuaud, Daniel, "Un Accueil chaleureux ", La Liberté, vol. 95, n 27, 2008, p. 27.

Bahuaud, Daniel, «Bien fêter la rentrée », La Liberté, vol. 95, n 25, 2008, p. 21.

Bahuaud, Daniel, "Brave Charles!", La Liberté, vol. 92, n 39, 2006, p. 22.

Bahuaud, Daniel, "Bravo les championnes ", La liberté, vol. 92, $\mathrm{n}^{\circ} 48$, 2006, p. 22.

Bahuaud, Daniel, "La Discipline sans contrainte ", La Liberté, vol. 95, $\mathrm{n}^{\circ} 22,2008$, p. 23.

Bahuaud, Daniel, "Un Esprit de gagnant ", La Liberté, vol. 92, n 45, 2006, p. 24. 
Bahuaud, Daniel, «L'Étoffe des héros ", La Liberté, vol. 92, n 43, 2006, p. 17.

Bahuaud, Daniel, "L'Excellence à l'honneur ", La Liberté, vol. 92, 2006, $\mathrm{n}^{\circ} 39$, p. 23.

Bahuaud, Daniel, "La Francisation en évolution ", La Liberté, vol. 95, $\mathrm{n}^{\circ} 24,2008$, p. 21.

Bahuaud, Daniel, «Un Talent démasqué », La Liberté, vol. 92, n 40, 2006, p. 25.

Bayart, Jean-François, L'Illusion identitaire, Paris, Fayard, coll. "L'espace du politique ", 1996, $306 \mathrm{p}$.

Bayart, Jean-François et Jean-Pierre Warnier (dir.), Matière à politique, le pouvoir, le corps, les choses, Paris, Karthala, coll. "Recherches internationales ", 2004, $256 \mathrm{p}$.

Braudel, Fernand, L'Identité de la France, "Espace et histoire ", tome I, Paris, Flammarion, coll. "Champs », 1990 [1986], 1181 p.

Breton, Philippe, La Parole manipulée, Paris, Éditions La Découverte, coll. "Essais ", 2000 [1997], 221 p.

Bruner, Jerome, Actual Minds, Possible Worlds, Cambridge (Mass.): Harvard University Press, 1986, $201 \mathrm{p}$.

Bruner, Jerome, Acts of Meaning, Cambridge (Mass.), Harvard University Press, 1990, $181 \mathrm{p}$.

Constalat-Founeau, Anne-Marie, Identité sociale et dynamique représentationnelle, Rennes, Presses universitaires de Rennes, coll. " Didactique et psychologie sociale ", 1997, 140 p.

Dubar, Claude, La Crise des identités. L'interprétation d'une mutation, Paris, Presses universitaires de France, 2000, 239 p.

Ehrenberg, Alain, La Fatigue d'être soi: dépression et société, Paris, Odile Jacob, 1998, $318 \mathrm{p}$.

Erickson, Erik Homburger, Childhood and Society, New York, WW Norton, 1963, 445 p.

Erickson, Erik Homburger, Identity, Youth and Crisis, New York, WW Norton, 1968, 336 p.

Esquenazy, Jean-Pierre, L'Écriture de l'actualité. Pour une sociologie du discours médiatique, Grenoble, Presses universitaires de Grenoble, coll. "Communication en plus ", 2002, 183 p.

Filioux, Jean-Claude et Gilles Billotte, Analyse d'un récit de vie. L'histoire d'Annabelle, Paris, Presses universitaires de France, 2005, 169 p.

Geertz, Clifford, The Interpretation of Culture and Selected Essays, New York, Basic Books, 1973, 470 p. 
Giddens, Anthony, Modernity and Self-Identity. Self and Society in the Late Modern Age, Cambridge, Polity Press, 1991, 264 p.

Goffman, Erving, Asiles. Etudes sur la condition sociale des malades mentaux, trad. de l'anglais par Liliane Lainé, Paris, Éditions de Minuit, coll. « Le sens commun ", 1968 [1961], 452 p.

Goffman, Erving, La Présentation de soi. La mise en scène de la vie quotidienne I, trad. de l'anglais par Alain Accardo, Paris, Éditions de Minuit, coll. "Le sens commun ", 1973 [1959], 256 p.

Goffman, Erving, Les Rites d'interaction, trad. de l'américain par Alain Kihm, Paris, Éditions de Éditions de Minuit, coll. «Le sens commun ", 1974 [1967], 240 p.

Goffman, Erving, Stigmate. Les usages sociaux des handicaps, trad. de l'anglais par Alain Kihm, Paris, Éditions de Minuit, coll. «Le sens commun », 1975 [1963], $176 \mathrm{p}$.

Habermas, Jurgen, Connaissance et intérêt, trad. française par Gérard Clémençon, Paris, Gallimard, coll. "Tel », 1991 [1979], 392 p.

Hall, Stuart Paul (dir.), Questions of Cultural Identity, London, Thousand Oaks, 1996, 198 p.

Kaufmann, Jean-Claude, «L'Identité, une nouvelle religion? », dans Sylvie Gruszow (dir.), L'Identité: qui suis-je ?", Paris, Éditions Le Pommier, 2006, p. 17-29.

Kaufmann, Jean-Claude, L'Invention de soi. Une théorie de l'identité, Paris, Hachette Littératures, coll. «Pluriel/sociologie », 2006 [2004], 352 p. Laplanche, Jean et Jean-Bertrand Pontalis, Vocabulaire de la psychanalyse, Paris, Presses universitaires de France, coll. «Bibliothèque de psychanalyse ", $523 \mathrm{p}$.

Lavoinne, Yves, Le Langage des médias, Grenoble, Presses universitaires de Grenoble, coll. "Communication en plus ", 1999, 142 p.

Lejeune, Philippe, Je est un autre: l'autobiographie, de la littérature aux médias, Paris, Éditions du Seuil, coll. "Poétique », 1980, 332 p.

Lipiansky, Edmond-Marc, Isabelle Taboada-Léonetti et Ana Vasquez, "Introduction à la problématique de l'identité », dans Carmel Camilleri et al. (dir.), Stratégies identitaires, Paris, Presses universitaires de France, coll. "Psychologie d'aujourd'hui ", 1990, 232 p.

Lipovetsky, Gilles, Le Bonheur paradoxal. Essai sur la société d'hyperconsommation, Paris, Gallimard, coll. "Folio/essais », 2009 [2006], 466 p.

Lochard, Guy et Henri Boyer, La Communication médiatique, Paris, Éditions du Seuil, coll. " Mémo », 1998, 96 p.

Martin, Denis-Constant, "Écarts d'identité, comment dire l'autre en politique ? ", dans Denis-Constant Martin (dir.), L'Identité en jeu: 
pouvoirs, identifications, mobilisations, Paris, Éditions Karthala, coll. "Recherches internationales ». 2010, p. 13-134.

Merton, Robert King, Social Theory and Social Structure, New York, Free Press, $702 \mathrm{p}$.

McKenzie, William James, Political Identity, Harmondsworth, Penguin, 1978, 239 p.

Meyer, Michel, Le Philosophe et les passions. Esquisse d'une histoire de la nature humaine, Paris, Presses universitaires de France, coll. "Quadrige », 2007, 408 p.

Molly, Andrew et al. (dir.), The Uses of Narrative: Exlorations in Sociology, Psychology and Cultural Studies, New Brunswick, Transaction Publishers, 2004, 202 p.

Muchembled, Robert, Culture populaire et culture des élites dans la France moderne (XV-XVIII $)$, Paris, Flammarion, 2002 [1978], 398 p.

Muchembled, Robert, L'Invention de l'homme moderne. Culture et sensibilités en France du XV au XVIII siècle, Paris, Fayard, coll. "Pluriel ", 1994 [1988], 517 p.

Prost, Antoine, "Frontières et espaces du privé ", dans Philippe Ariès et Georges Duby (dir.), Histoire de la vie privée, "De la Première Guerre mondiale à nos jours », tome V, Paris, Editions du Seuil, coll. « Points/ histoire", 1999 [1985], p. 14-132.

Ricœur, Paul, L'Idéologie et l'utopie, Paris, Éditions du Seuil, coll. « Points/ essais ", 1997, $410 \mathrm{p}$.

Ricœur, Paul, La Mémoire, l'histoire, l'oubli, Paris, Éditions du Seuil, coll. "Points », 2003 [2000], 675 p.

Ricœur, Paul, Parcours de la reconnaissance. Trois études, Paris, Gallimard, coll. «Folio/essais », 2007 [2004], 435 p.

Ricœur, Paul, Soi-même comme un autre, Paris, Éditions du Seuil, coll. "L’ordre philosophique ", 1990, 424 p.

Ricœur, Paul, Temps et récit, volume I, "L'intrigue et le récit historique ", Paris, Éditions du Seuil, coll. "L'ordre philosophique ", 1983, 322 p.

Ricœur, Paul, Temps et récit, volume II, «La configuration dans le récit de fiction », Paris, coll. Éditions du Seuil, «L'ordre philosophique », 1991 [1984], $300 \mathrm{p}$.

Ricœur, Paul, Temps et récit, volume III, "Le temps raconté ", Paris, Gallimard, Éditions du Seuil, coll. "L'ordre philosophique », 1991 [1985], $374 \mathrm{p}$.

Sauvageot, Anne, Voirs et Savoirs. Esquisse d'une sociologie du regard, Paris, Presses universitaires de France, coll. "Sociologie d'aujourd'hui ", 1994, 249 p. 
Sfez, Lucien, Critique de la communication, nouvelle édition entièrement refondue et augmentée, Paris, Seuil, 1992 [1988], coll. « Point », 527 p.

Singly, François de, Libres ensemble. L'Individualisme dans la vie commune, Paris, Nathan, 2000, coll. " Essais et recherches ", 253 p.

Singly, François de, Les Uns avec les autres: quand l'individualisme crée du lien, Paris, Armand Colin, coll. "Individu et société ", 2003, 267 p.

Singly, François de et Daniel Bertaux, Daniel (dir.), Le Récit de vie, Paris, Armand Colin, coll. "L'enquête et ses méthodes ", 2005, 126 p.

Somers, Margaret, "The Narrative Constitution of Identity: A Relational and Network Approach ", Theory and Society, vol. 23, n 5, 1994, p. 605-649.

Stets, Jan et Peter Burke, "A Sociological Approach to Self and Identity ", dans Marc R. Leary et June Price Tangney, (dir.), Handbook of Self and Identity, New York, Gilford Press, 2002, p. 99-114.

Tap, Pierre, Identités collectives et changements sociaux: production et affirmation de l'identité, tome 2, Toulouse, Privat, coll. "Sciences de l'homme ", 1980, $456 \mathrm{p}$.

Tap, Pierre (dir.), Identités individuelles et personnalisation: production et affirmation de l'identité, tome 1, Toulouse, Privat, coll. "Sciences de l'homme ", 1980, $412 \mathrm{p}$.

Taylor, Charles, Le Sources du moi. La formation de l'identité moderne, trad. française par Charlotte Melançon, Montréal, Boréal, coll. "Compact ", 2003 [1992], $712 \mathrm{p}$.

Tap, Pierre et Ami Gutman, Multiculturalisme: différence et démocratie, Paris, Flammarion, coll. "Champs », 1997, 144 p.

Touraine, Alain, Un Nouveau paradigme. Pour comprendre le monde d'aujourd'hui, Paris, Fayard, coll. "Biblio/essais », 2008 [2006], 410 p. 